\title{
Association of telomere length in peripheral blood leukocytes with the presence of atrial fibrillation in elderly male
}

\author{
Chuanbin Liu ${ }^{1}$, Jing Bai ${ }^{1}$, Qing $\mathrm{Dan}^{2}$, Xue Yang${ }^{2}$, Zihao $\mathrm{Fu}^{1}, \mathrm{Xu} \mathrm{Lu}^{1}$, Xiaoye Xie ${ }^{2}$, Li \\ $\mathrm{Fan}^{2}$, Feng $\mathrm{CaO}^{2}$, yang $\mathrm{li}^{2}$, and Jianwei $\mathrm{Liu}^{2}$ \\ ${ }^{1}$ Medical School of Chinese PLA \\ ${ }^{2}$ Chinese PLA General Hospital
}

October 9, 2020

\begin{abstract}
Objective: Telomeres gradually shorten and the incidence of atrial fibrillation (AF) gradually increases with age. However, the association of telomere length with AF is still controversial. This study aims to determine the correlation between the leukocyte telomere length (LTL) and the presence of AF. Methods: This study recruited 96 AF male patients and 97 healthy male controls (aged [?]60 years) Anthropometric, clinical and laboratory analysis were performed on all subjects. Blood LTL was detected by quantitative real-time PCR assay. PGC- $1 \alpha$ concentration was evaluated by ELISA method. The association between LTL and AF was analyzed by simple and multivariate logistic regression. Results: LTL in AF patients was significantly shorter than controls $(\mathrm{P}<0.001)$. Logistic regression analysis confirmed that LTL was inversely associated with the presence of AF (OR 0.428, 95\% confidence interval [CI]: 0.268-0.684; $\mathrm{P}<0.001$ ). Furthermore, we conducted a subgroup analysis of different ages and different types of AF and found that LTL was negatively correlated with age $(\mathrm{r}=-0.148, \mathrm{P}=0.040)$, and there was no statistical difference among different types of AF. We found that the telomere-associated molecule serum PGC-1 $\alpha$ concentration was also negatively related to AF (OR 0.991, 95\% CI: 0.985-0.997; P = 0.004) . Conclusion: LTL and serum PGC- $1 \alpha$ concentration are inversely correlated with the presence of AF in elderly male.
\end{abstract}

\section{Hosted file}

Main document.pdf available at https://authorea.com/users/365864/articles/485801association-of-telomere-length-in-peripheral-blood-leukocytes-with-the-presence-ofatrial-fibrillation-in-elderly-male 